'State University of Piauí - UESPI, School of Dentistry, Department of Clinical Dentistry, Area of Integrated Clinic, Parnaíba, PI, Brazil.
Corresponding author: Ana de Lourdes Sá de Lira Universidade Estadual do Piauí, Faculdade de Odontologia Rua Senador Joaquim Pires 2076 Ininga.

Fone (86) 999595004

CEP: 64049-590

Teresina-PI-Brasil

email: anadelourdessl@hotmail.com

Editor: Dr Altair A. Del Bel Cury

Received: September 12, 2020

Accepted: February 17, 2021

\section{Prevalence and predisponent factors of molar-incisor hypomineralization in primary dentition}

\author{
Ana de Lourdes Sá de Lira ${ }^{1, *} \mathbb{D}$, Francisca Janiele de \\ Sousa ${ }^{1}$, Francisco Dário Carvalho de Sousa ${ }^{1}$, Maria \\ Karen Vasconcelos Fontenele ${ }^{1}$, Carlos Kelvin Campos \\ Ribeiro' ${ }^{1}$, Luiz Eduardo Gomes Ferreira ${ }^{1}$
}

Aim: To evaluate the prevalence and predisposing factors for hypomineralization of second molars in children in primary dentition. Methods: A questionnaire was applied to parents to analyze predisposing factors and to assist in the diagnosis of hypomineralization in children between 2 and 6 years old, followed by an intraoral examination based on indices of non-fluorotic enamel defects in the primary dentition, according to the "Modified Index DDE" to determine demarcated opacity and HSPM presence / severity index to assess hypomineralization. Children from public and private schools were dived into two groups: if they presented HSPM-Group 1 (G1) and if they did not have HSPM-Control group (CG). Results: The most frequent predisposing factors associated with the child were Illness in the first year of life $(X 2=6.49 ; p=0.01)$ and antibiotic use in the first year of life $(X 2=41.82 ; p=0.01)$. The factors associated with the mother were hypertension $(X 2=9.36 ; p=0.01)$, infections during pregnancy $(X 2=14.80 ; p=0.01)$ and alcohol consumption during pregnancy $(X 2=97.33 ; p=0.01)$. There was a prevalence of $3.9 \%$ of HSPM in 14 children, with statistical difference regarding gender $(\mathrm{X} 2=4.57 ; \mathrm{p}<0.05)$, with boys presenting a higher frequency. In G1 hypomineralization was of the type with demarcated opacity, with more prevalent characteristics the yellowish spot, with moderate post-eruptive fracture and acceptable atypical restorations. All lesions were located in the labial region with $1 / 3$ of extension. Conclusion: The prevalence of HSPM in children between 2 and 6 years old was $3.9 \%$, with a predominance in males, with tooth 65 being the most affected. There was an association between HSPM and infection in the first year of life, as well as the use of antibiotics and sensitivity in the teeth affected by the lesion. There was an association between HSPM and hypertension, infection and mothers' alcohol use during pregnancy.

KEYWORDS: Tooth demineralization. Dental enamel. Amelogenesis. 


\section{Introduction}

Molar-incisor hypomineralization ( $\mathrm{MIH})$ is a defect of systemic origin in the dental enamel that affects the first permanent molars and is often associated with the incisors. It is characterized by qualitative defects in dental tissues and is visually identified by the change in enamel translucency ${ }^{\top}$. It is a clinical condition that appears in the permanent dentition, but that can be related to hypomineralization in the primary dentition. Several studies attempt to investigate factors potentially involved with the occurrence of $\mathrm{MIH}$, however the findings are variable and inconclusive ${ }^{2,3}$.

The etiology remains unknown, although it is related to environmental factors that are associated with systemic conditions during the prenatal period (last three months of pregnancy) and during the perinatal and postnatal periods. These factors may include low birth weight, congenital heart disease and use of continuous medication to treat systemic disease. Common conditions in the first 3 years of age, such as diseases of the upper respiratory tract, asthma, otitis, tonsillitis, gastrointestinal diseases, malnutrition, chickenpox, measles and rubella, also seem to be associated with the occurrence of $\mathrm{MIH}^{4-6}$.

Due to the temporal association between the mineralization of the crowns of the first permanent molars and the second deciduous molars, they may also be affected by hypomineralization if any etiological factor, such as some systemic disorder, acts during this stage of development, especially during the period prenatal and perinatal ${ }^{7}$.

The hypomineralization of second primary molars (HSPM) is similar to MIH with respect to both asymmetric distribution and post-eruptive structural losses and atypical restorations. The researchers suggest that the presence of HSPM in the primary dentition poses a risk for the presence of $\mathrm{MIH}$. The explanation for this association is probably related to the etiological factors of developmental defects in the enamel, which are the same in both dentitions. The intensity and duration of injuries that occur in late pregnancy and in the early perinatal period can affect both dentitions ${ }^{8}$.

The characteristics of HSPM are the same as those of MIH: opaque spots that vary in tone between white, yellow and brown, break post-eruptive enamel, atypical restorations and / or extensive caries with opacities in the margins, sensitivity, which can affect one to four second molars ${ }^{7}$.

In the same patient it can be presented in a different way, being possible to observe a slight opacity in one second molar, and great structural losses in another, mainly the contralateral one, with the occurrence of fractures soon after the tooth eruption ${ }^{8,9}$.

Because the characteristics and clinical consequences are similar and the intention to identify the etiological factors involved in this enamel alteration, the epidemiological knowledge of the distribution of this defect is necessary. This research is justified because there is no study that reports the prevalence of HSPM in the city where the search was conducted.

It is believed that there is a low prevalence of HSPM in the primary dentition and ignorance of parents and / or guardians about the damage of the injury in the child's oral 
health quality. In addition, the presence of HSPM can be considered a predictive factor for $\mathrm{MIH}$, although the absence of this defect in the primary dentition does not exclude the future appearance of $\mathrm{MIH}$.

The aim of this research was to evaluate the prevalence and predisposing factors for hypomineralization of second molars in children in primary dentition.

\section{Material and Methods}

This study was approved by the Research Ethics Committee of the State University of Piauí - CEP / UESPI under the number: 3,289,708. It was a cross-sectional study, whose sample calculation was based on the target audience: preschool students from the city of Parnaíba-PI, attending in public and private schools in 2019. The sample size calculation was according to a survey conducted by the Brazilian Institute of Geography and Statistics, which showed an estimated 15.000 children living in the city of Parnaíba-PI aged 2 to 6 years.

Thus, the necessary sample size was 360 participants. It was decided to increase the exhibition to 365 , assuming the possibility of giving up participation in the research. In fact, 5 children were excluded from the survey because their parents answered the questionnaire incompletely, making a total of 360 participants.

This minimum number of participants was considered sufficient considering the proposed analyzes, the sample error of $5 \%$, in addition to the $95 \%$ confidence level, indicating that the probability of the error made by the research did not exceed $5 \%{ }^{10}$.

The researchers were provided with a letter of consent from the principals of the schools, chosen by lot, being a private and a public institution, in the municipality of Parnaíba-Piauí, which authorized the development of the research after the ethical approval of the Research Ethics Committee of the State University of Piauí - CEP / UESPI, from August to December 2019.

Inclusion criteria were children in primary dentition, randomly included, between 2 and 6 years of age, who had four primary second molars fully erupted at the time of examination whose parents or guardians and children accepted the research. As exclusion criteria, children under 2 years old, as the primary dentition would not yet be fully formed, and those over 6 years old, because they would already be in mixed dentition, those pre-schoolers with a syndrome or special need, who did not tolerate the dental examination or who had fixed orthodontic appliance.

In order to standardize the diagnosis of HSPM, clinical training of examiners was carried out in the children's clinic of Clinic School of Dentistry (CSD) of of the State University of Piauí (UESPI) Campus of Parnaíba. To measure the intra and inter-examiner diagnostic reproducibility, $10 \%$ of the total sample was doubly verified by two examiners, with the Kappa coefficient for intra and inter-examiner agreement being 0.87 and 0.84 , respectively.

Before data collection, a pilot study was carried out with 50 children from municipal schools who did not participate in the sample, to evaluate the methods and it was found that there was no need to make changes to the methodology initially proposed. 
The parents / guardian of the children answered the questionnaire to help diagnose hypomineraization (Figure 1), after having attended a lecture at the school on the topic. Using an age-compatible language, the children received an explanation of how the research would be conducted. In a second moment in the school environment, the children were examined. The children were seated in a school chair, with their head positioned on the examiner's lap.

1.Child gender: female () male ()

2. Age of child: 2 years () 3 years () 4 years () 5 years () 6 years ()

3. Race: white () black () brown ()

4. School: public () private ()

5. During pregnancy:

Hypertension: yes () no ( )

Diabetes: yes ( ) no ( )

Infections: yes ( ) no ( )

Cigarette consumption:

Alcohol consumption:

Use of medication:

6. In relation to childbirth:

yes ( ) no ( )

yes ( ) no ()

yes () no () if so, which one?

normal () cesarean ( )

complications: yes () no()

premature childbirth: yes () no()

7. In relation to child :

breathing problem at birth: yes ( ) no ( )

postpartum hospitalization: yes( ) no ()

premature baby: yes () no ()

need for incubator: yes () no ()

born with jandice/bluish: yes( ) no( )

duration of breastfeeding:

illness in the first 3 years of life:yes () no() if so, which one?

high fever often in the first 3 years of life:yes () no

antibiotic use in the first 3 years of live: yes ( ) no ( )

if so, how often and which most used

medication use for more than 30 days in the first 3 years of live:

yes ( ) no ( ) if so, which one?

tooth sensitivite: yes ( ) no ( )

stained tooth: yes ( ) no ( )

Figure 1. Evaluation of hypomineralization of second primary molars (HSPM).

The clinical examination was carried out under natural light, in a school environment, by two specially calibrated examiners, previously calibrated, with the aid of a wooden spatula, mouth mirror and explorer probe. After cleaning and drying the teeth with sterile gauze, a complete inspection was performed, based on the following indices: non-fluorotic enamel defects in the primary dentition, according to the "Modified Index DDE", assessing the coloring of the demarcated opacity ( white, yellow or brown), extension of defect (less than $1 / 3$ of a tooth surface; at least $1 / 3$ but less than $2 / 3$; and at least $2 / 3$ of the tooth surface), location (labial/buccal, lingual/palatal or occlusal), 
if there is presence of post-eruptive fracture (light, moderate or severe) and atypical restoration (acceptable/no acceptable) (Figure 2) ${ }^{11}$. According to the characteristics of the enamel defects, children were classified as having or not hypomineralization. Those who presented HSPM were named group 1 (G1) and those who did not present the lesion constituted the control group (CG) in both public (A1) and private schools (A2). The observations were transcribed to a standardized clinical record according to the established codifications.

\begin{tabular}{lccc}
\hline Dental characteristic & Code & Code & Code \\
\hline Demarcated opacity & White stain (a) & Yellow stain (b) & Brown stain (c) \\
\hline Post-eruptive fracture & Light (d) & Moderate (e) & Severe (f) \\
\hline Atypical restoration & Acceptable (g) & No aceptable (h) & \\
\hline
\end{tabular}

Figure 2. Judgment used in the diagnosis of HSPM based on severity.

During the examination, it was possible to identify the need to apply fluoride to minimize tooth sensitivity or restorative treatment in the presence of irregularities in the enamel structure. In such situations the children were referred to the CSD of UESPI for assistance and follow-up at the pediatric dentistry clinic.

The results were stored in the Excel Windows 2007 Microsoft ${ }^{\circledR}$ database and displayed in graphs and tables for better interpretation and discussion. With SPSS, in its version 25, descriptive and association statistical tests were performed, with chi square, in addition to comparison of means using the t test. All considering the significance level $p$ value of 0.05 .

\section{Results}

At first, we sought to know the prevalence of HSPM. Based on the total sample of 360 children, a prevalence of $3.9 \%$ was observed, that is, 14 children, 8 of whom were brown, 5 white and 1 black. Of this total, 11 had lesions on two teeth, with $43 \%(n=6)$ of children having teeth 55 and 65 (upper deciduous second molars) affected, followed by those with teeth 75 and 85 (lower deciduous second molars) $(36 \% ; n=5)$, and finally, those with only tooth 65 affected $(21 \% ; n=3)$.

Of the children in G1, 11 were male and 3 were female, with 6 studying in public schools (A1) and 8 in private schools (A2). On the other hand, those in CG, 190 were male and 156 female, with 171 studying in A1 and 175 in A2 (Figure 3).

According to Table 1, 9 children presented tooth 65 as the most affected by hypomineralization, followed by 55 in 6 children. HSPM presented as demarcated opacity, with the most prevalent characteristics being the type of yellowish spot, with moderate post-eruptive fracture, but with atypical restorations acceptable. 


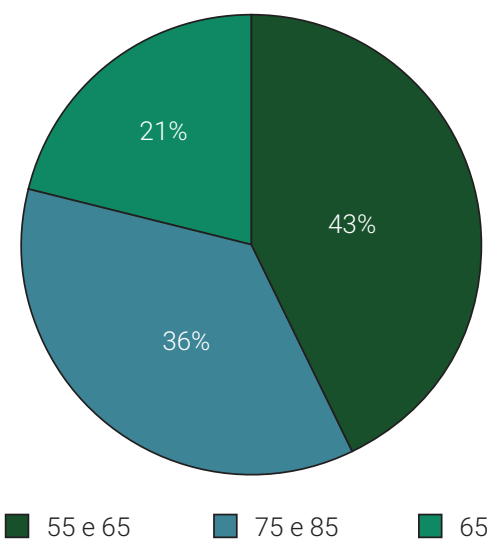

Figure 3. Comparison between $\mathrm{G} 1$ and GC regarding gender and school.

Table 1. Teeth affected and characteristics of HSPM in children.

\begin{tabular}{lcccc}
\hline Tooth & Number of children & Type of stain & Post -eruptive fracture & Atypical restoration \\
\hline 55 & 6 & yellowish & moderate & acceptable \\
\hline 65 & 3 & yellowish & moderate & acceptable \\
\hline 75 & 2 & yellowish & moderate & acceptable \\
\hline 85 & 4 & yellowish & moderate & acceptable \\
\hline 65 & 5 & brown & moderate & not acceptable \\
\hline 65 & 1 & white & light & acceptable \\
\hline 75 & 3 & brown & light & acceptable \\
\hline 85 & 1 & brown & moderate & not acceptable \\
\hline
\end{tabular}

According to the frequency distribution of the affected teeth, there was no statistically significant difference between them, based on the chi-square test ( $p>0.05)$.

The t test was performed to compare the mean breastfeeding time of G1 and CG, and it was possible to verify that there was a statistically significant difference $(t=2.66$; $p=0.01$ ), with higher averages of time for the CG (13.3 months) when compared to G1 (10.5 months). As for age, there was no difference between groups ( $p>0.05)$. Finally, it is highlighted that $100 \%$ of children in $\mathrm{G} 1$ have tooth sensitivity.

In sequence, it was possible to observe, from Figure 4, the difference in the frequency of HSPM between gender and children's school.

From the data and the statistical calculation of the chi-square, it was observed that in the children of the CG there were no differences, however in the children of G1 there was a difference regarding gender $(X 2=4.57$; $p<0.05)$, having boys showed a higher prevalence. Table 2 shows the distribution of the predisposing factors for children. 


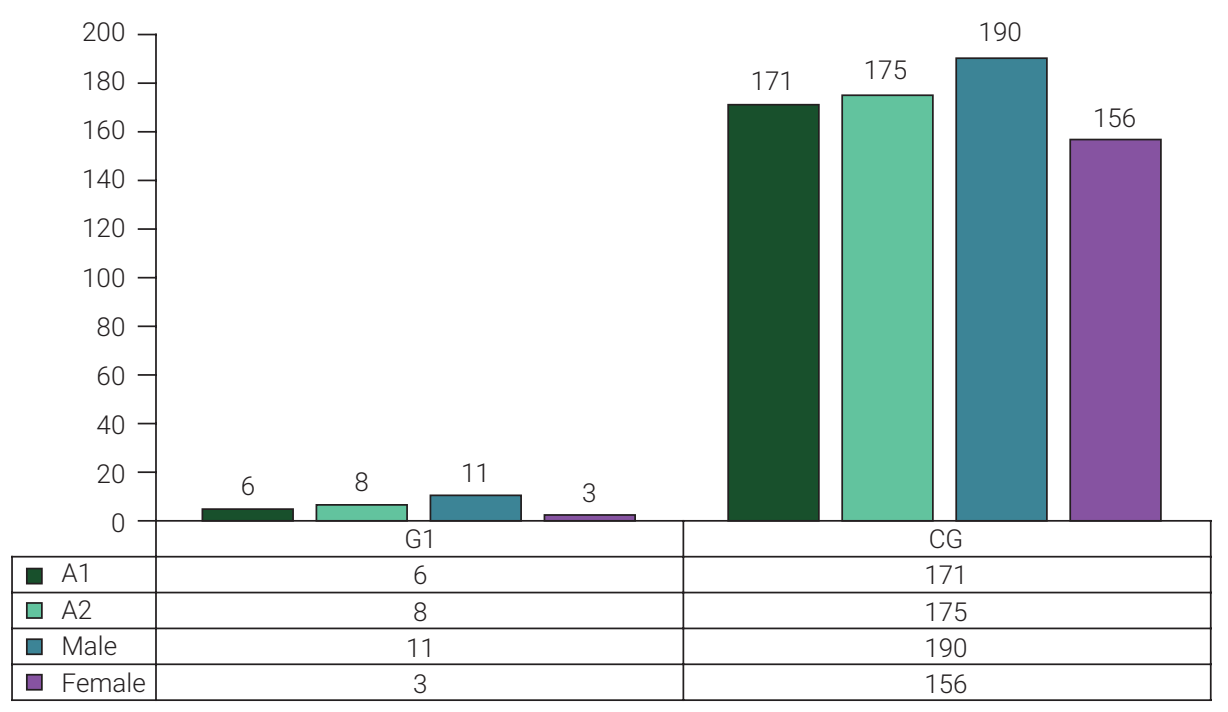

Figure 4. Difference in the frequency of HSPM between gender and children's school.

Table 2. Distribution of the predisposing factors for children from CG and G1.

\begin{tabular}{|c|c|c|c|c|c|}
\hline Predisposing factors/Child & & CG & G1 & Total & $\begin{array}{c}\mathrm{X}^{2} \\
p \text { valor }\end{array}$ \\
\hline \multirow{2}{*}{ Breathing problem at birth } & No & 319 & 14 & 333 & 1.18 \\
\hline & Yes & 27 & 0 & 27 & 0.28 \\
\hline \multirow{2}{*}{ Neonatal hospitalization } & No & 280 & 11 & 291 & 0.05 \\
\hline & Yes & 66 & 3 & 69 & 0.83 \\
\hline \multirow{2}{*}{ Premature baby } & No & 334 & 14 & 348 & 0.51 \\
\hline & Yes & 12 & 0 & 12 & 0.48 \\
\hline \multirow{2}{*}{ Birth weight } & $<2.5 \mathrm{Kg}$ & 102 & 244 & 346 & 0.42 \\
\hline & $>2.5 \mathrm{Kg}$ & 3 & 11 & 14 & 0.52 \\
\hline \multirow{2}{*}{ Need for incubator } & No & 313 & 14 & 327 & 1.47 \\
\hline & Yes & 33 & 0 & 33 & 0.23 \\
\hline \multirow{2}{*}{ Born with jaundice/bluish } & No & 268 & 11 & 279 & 0.10 \\
\hline & Yes & 78 & 3 & 81 & 0.92 \\
\hline \multirow{2}{*}{ Illness in the first year of life } & No & 111 & 0 & 111 & 6.49 \\
\hline & Yes & 235 & 14 & 249 & $0.01 *$ \\
\hline \multirow{2}{*}{ High fever often in the first year of life } & No & 331 & 14 & 345 & 0.63 \\
\hline & Yes & 15 & 0 & 15 & 0.42 \\
\hline \multirow{2}{*}{ Antibiotic use in the first year of life } & No & 267 & 0 & 267 & 41.82 \\
\hline & Yes & 79 & 14 & 93 & $0.01 *$ \\
\hline \multirow{2}{*}{ Tooth sensitivity } & No & 345 & 0 & 345 & $335.03 *$ \\
\hline & Yes & 1 & 14 & 15 & $0.01 *$ \\
\hline
\end{tabular}

Foot note: ${ }^{*} p<0,05$ 
Based on Table 2, with the frequency data, followed by the chi-square test, it was observed in G1 that there was an association between the presence of HSPM and the fact that the child had some disease in the first year of life $(p=0,01)$, as well as, with the use of some antibiotic ( $p=0.01)$ and sensitivity in the teeth affected by the lesion $(p=0.01)$. Table 3 shows the predisposing factors for HSPM for mothers.

Table 3. Distribution of predisposing factors for mothers of children in G1 and CG.

\begin{tabular}{|c|c|c|c|c|c|}
\hline Predisposing factors/Mother & & CG & G1 & Total & $\begin{array}{c}X^{2} \\
p \text { valor }\end{array}$ \\
\hline \multirow{2}{*}{ Hypertension } & No & 312 & 9 & 321 & 9.36 \\
\hline & Yes & 34 & 5 & 39 & $0.01 *$ \\
\hline \multirow{2}{*}{ Diabetes } & No & 319 & 14 & 333 & 1.18 \\
\hline & Yes & 27 & 0 & 27 & 0.57 \\
\hline \multirow{2}{*}{ Infections during pregnancy } & No & 337 & 11 & 348 & 14.80 \\
\hline & Yes & 9 & 3 & 12 & $0.01 *$ \\
\hline \multirow{2}{*}{ Smoking during pregnancy } & No & 337 & 14 & 351 & 0.37 \\
\hline & Yes & 9 & 0 & 9 & 0.54 \\
\hline \multirow{2}{*}{ Alcohol consumption during pregnancy } & No & 343 & 8 & 351 & 97.33 \\
\hline & Yes & 3 & 6 & 9 & $0.01 *$ \\
\hline \multirow{2}{*}{ Use of medication during pregnancy } & No & 313 & 11 & 324 & 2.11 \\
\hline & Yes & 33 & 3 & 36 & 0.15 \\
\hline \multirow{2}{*}{ Type of childbirth } & Normal & 277 & 14 & 291 & 3.54 \\
\hline & Cesarean & 69 & 0 & 69 & 0.06 \\
\hline \multirow{2}{*}{ Complications during childbirth } & No & 322 & 14 & 326 & 1.04 \\
\hline & Yes & 24 & 0 & 24 & 0.31 \\
\hline \multirow{2}{*}{ Premature childbirth } & No & 334 & 14 & 348 & 0.50 \\
\hline & Yes & 12 & 0 & 12 & 0.48 \\
\hline
\end{tabular}

Foot note: ${ }^{*} p<0,05$

From Table 3, with the frequency data, followed by the chi-square test, it was possible to observe an association between the presence of HSPM in G1 and the fact that the mother was hypertensive $(p=0.01)$. That is, in five of the 14 children in $\mathrm{G} 1$, the mothers were hypertensive. In the same line of analysis, an association was observed between $\mathrm{G} 1$ and mothers who were affected by some infection during pregnancy $(n=3)(p=0.01)$ and those who consumed alcohol during pregnancy $(n=6)(p=0.01)$.

\section{Discussion}

Regarding the prevalence of $3.9 \%$ of HSPM in the present study, similar results were observed by some authors ${ }^{8,12}$ whose prevalence was $2.9 \%$. Other authors ${ }^{13-16}$ found varying prevalences, since they are in accordance with population characteristics, methodological differences between studies, sample size and children's age range. 
According to Elfrink et al. ${ }^{12}$ (2012) and Aine et al. ${ }^{17}$ (2000) there is a strong correlation between HSPM and MIH. In this research, most of the children $(n=6)$ had their upper deciduous second molars affected. One study evaluated the prevalence and association between HMI and HSPM and primary canines in Brazilian schoolchildren. It was found a prevalence for second molars of $6.48 \%$ and for deciduous canines $2.22 \%$. It is worth mentioning that according to Sé et al. ${ }^{18}$ (2017), children with hypomineralized second molars and primary canines are six times more likely to develop IMH.

In view of this fact, the 14 children who presented HSPM should be monitored until the eruption of the first molars and permanent incisors because they are highly likely to present HMI, since the enamel mineralization phase of these teeth occurs in the same period ${ }^{15,19}$.

In this study, it was observed that in children in $\mathrm{G} 1$ there was a significant difference in terms of gender $(X 2=4.57 ; p<0.05)$, with boys having a higher prevalence of HSPM. Disagreeing with the studies by Kemoli20 (2008) and Jeremias et al. ${ }^{21}$ (2013) whose frequency was higher in females, $76 \%$ and $62 \%$ respectively, and by Subramaniam et al. ${ }^{22}$ (2016) who did not find a gender predilection.

The $t$ test was performed to compare the average breastfeeding time between G1 and CG, verifying that there was a statistically significant difference $(t=2.66$; $p=0.01)$, with higher averages of time for CG (13,28 months) when compared to $G 1$ (10.50 months). Paradoxically, research carried out by some authors ${ }^{23,24}$ suggested that children who had been exposed to dioxin through prolonged breastfeeding had a higher risk of developing hypomineralization.

In the survey, it was highlighted that $100 \%$ of children with hypomineralization had stained teeth with demarcated opacity, tooth wear with loss of enamel, increased susceptibility to caries, since all teeth were restored and tooth hypersensitivity. According to the literature, hypomineralization has a greater impact on the quality of life of affected children, whether due to pain, sensitivity to thermal stimuli or the aesthetic appearance of opacities in teeth ${ }^{25-27}$.

In this research it was possible to observe that the presence of hypomineralization was associated with the fact that the child had had a disease in the first year of life. According to Elfrik et al..$^{8}(2015)$, the first year of life is a critical period for the formation of the crown of permanent molars and incisors, and thus for the development of HMI. Infectious conditions with repeated episodes of high fever can be considered as risk factors for the development of hypomineralization, as well as prenatal complications, premature birth, childhood illnesses accompanied by high fever in the first three years of life, as shown by our search.

The current study showed that all children in G1 used some antibiotic, the most frequent of which were Amoxicillin and Azithromycin. Following the same line, Faustino-Silva et al. ${ }^{28}(2020)$ in their research showed that there was an association between children with dental enamel defects (DED) and those who used antibiotics, with amoxicillin being the most commonly prescribed medication. A similar fact was observed by Wuollet et al. ${ }^{27}$ (2016) and Lopes-Fatturi et al. ${ }^{29}$ (2019) when they found that children with hypomineralization sought more care for infectious dis- 
eases, received more penicillin in the first year of life, or amoxicillin until the 3 years, than children without hypomineralization.

It was observed in this research the association between hypomineralization and the fact that the mother had hypertension, some infection, or consumed alcohol during pregnancy. Corroborating the study by Lopes-Fatturi et al. ${ }^{29}$ (2019) when they observed that smoking use, presence of hypertension, complications during childbirth are associated with a higher prevalence of hypomineralized deciduous second molars (HSPM).

The causes of hypomineralization in teeth have not been fully clarified since there are several hypotheses such as diseases of the mother during pregnancy, premature birth, systemic diseases (mainly respiratory), exposure to products and medications that are considered to be risk factors ${ }^{7,30}$.

However, according to the literature, hypomineralization is defined as a qualitative enamel defect caused by a disturbance during initial calcification and / or during maturation, being an important risk factor for caries in hypomineralized deciduous and permanent molars and that the presence of HSPM can be a precursor to $\mathrm{MIH}$, and the need for monitoring and controlling injuries is essential. It is worth mentioning that the absence of this defect in the primary molars does not exclude the appearance of $\mathrm{MIH}^{7,31}$.

In conclusion, the prevalence of HSPM in children between 2 and 6 years old was $3.9 \%$, with predominance in males, with tooth 65 being the most affected. HSPM presented as demarcated opacity, with the most prevalent characteristics being the type of yellowish spot, with moderate post-eruptive fracture and acceptable atypical restorations. There was an association between HSPM and infection in the first year of life, as well as the use of antibiotics and sensitivity in the teeth affected by the lesion. There was an association between HSPM and hypertension, infection and mothers' alcohol use during pregnancy.

\section{References}

1. Weerheijm KL, Duggal M, Mejare I, Papagiannoulis L, Koch G, Martens LC, et al. Judgement criteria for molar incisor hypomineralisation $(\mathrm{MIH})$ in epidemiologic studies: a summary of the European meeting on MIH held in Athens, 2003. Eur J Paediatr Dent. 2003;4(3)110-3.

2. Jalevik B. Prevalence and diagnosis of molar-incisor-ypomineralization (MIH): A systematic review. Eur Archs Paediatr Dent. 2010;11(2):59-64. doi: 10.1007/BF03262714.

3. Farias L, Laureano ICC, Alencar CRB, Cavalcanti AL. [Molar incisor hypomineralization: etiology, clinical characteristics and treatment]. Rev Cienc Med Biol. 2018;17(2):211-9. Portuguese. doi: 10.9771/cmbio.v17i2.27435.

4. Assunção CM, Girelli V, Sarti CS, Ferreira ES, Araujo FB, Rodrigues JA. [Molar incisor hypomineralization (MIH): case report and restorative treatment follow-up]. Rev Assoc Paul Cir Dent. 2014;68(4):346-50. Portuguese.

5. Weerheijm KL. Molar incisor hypomineralization (MIH). Eur J Paediatr Dent. 2003;4(3):115-20.

6. Fragelli CM, Jeremias F, Feltrin de Souza J, Paschoal MA, Cordeiro RCL, Santos-Pinto L. Longitudinal evaluation of the structural integrity of teeth affected by molar incisor hypomineralization. Caries Res. 2015;49(4):378-83. doi: 10.1159/000380858. 
7. Negre-Barber A, Montiel-Company J, Boronat-Catalá M, Catalá-Pizarro M, Almerich JM. Hypomineralized second primary molars as predictor of molar incisor hypomineralization. Sci Rep. 2016;6(3):1-6. doi:10.1038/srep31929.

8. Elfrink MEC, Ghanim A, Manton DJ, Weerheijm KL. Standardised studies on molar incisor hypomineralization $(\mathrm{MIH})$ and hypomineralized second primary molars (HSPM): a need. Eur Arch Paediatr Dent. 2015;16(3):247-55. doi: 10.1007/s40368-015-0179-7.

9. Mittal N, Sharma BB. Hypomineralised second primary molars: prevalence, defect characteristics and possible association with molar incisor hypomineralization in indian children. Eur Arch Paediatr Dent. 2015 Dec;16(6):441-7. doi: 10.1007/s40368-015-0190-z.

10. Luchesa CJ. Chaves Neto A. [Calculation of sample size in administration research]. Curitiba: Edição do autor; 2011. 27p. Portuguese.

11. Ghanim A, Elfrink M, Weerheijm K, Mariño R, Manton D. A practical method for use in epidemiological studies of enamel hpomineralization. Eur Arch Paediatr Dent. 2015;16(3):235-46. doi: 10.1007/s40368-015-0178-8.

12. Elfrink MEC, Ten Cate JM, Jaddoe VWV, Hofman A, Moll Ha, Veerkamp JSJ. Deciduous molar hypomineralization and molar incisor hypomineralization. J Dent Res. 2012 Jun;9(16):551- 5. doi: $10.1177 / 0022034512440450$.

13. Owen M, Ghanim A, Elsby D, Manton, D. Hypomineralized second primary molars: prevalence, defect characteristics and relationship with dental caries in Melbourne preschool children. Aust Dent J. 2007;63(1):72-80. doi:10.1111/adj.12567

14. Halalet F, Raslan N. Prevalence of hypomineralized second primary molars (HSPM) in Syrian preschool children. Eur Arch Paediatr Dent. 2020 Dec;21(6):711-7. doi: 10.1007/s40368-020-00520-2.

15. Sidhu N, Wang Y, Barrett E, Casas M. Prevalence and presentation patterns of enamel hypomineralization (MIH and HSPM) among paediatric hospital dental patients in Toronto, Canada: a cross-sectional study. Eur Arch Paediatr Dent. 2020;21(2):263-70. doi:10.1007/s40368-019-00477-x

16. Masumo R, Bardsen A, Astrom AN. Developmental defects of enamel in primary teeth and association with early life course events: a study of 6-36 month old children in Manyara, Tanzania. BMC Oral Health. 2013 May;13(21):1-11. doi: 10.1186/1472-6831-13-21.

17. Aine L, Backström MC, Mäki R, Kuusela AL, Koivisto AM, Ikonen RS, et al. Enamel defects in primary and permanent teeth in children born prematurely. J Oral Pathol Oral Med. 2000;29(8):403-9. doi:10.1034/j.1600-0714.2000.290806.x.

18. Sé MJSF, Ribeiro APD, Dos Santos-Pinto LAM, Loiola RC, Cordeiro R, Cabral RN, et al. Are hypomineralized primary molars and canines associated with molar-incisor hHypomineralization? Pediatr Dent. 2017 Nov;39(7):445-9.

19. Serna-Muñoz C, Ortiz-Ruiz AJ, Silva AP, Bravo-González LA, Vicente A. Second primary molar hypomineralization and drugs used during pregnancy and infancy. A systematic review. Clin Oral Invest. 2020 Mar;24(3):287-97. doi:10.1007/s00784-019-03007-7.

20. Kemoli AM. Prevalence of molar incisor hypomineralization in six to eight year-olds in two rural divisions in Kenya. East Afr Med J. 2008;85(10):514-9.

21. Jeremias F, de Souza JF, Silva CM, Cordeiro RC, Zuanon AC, Santos-Pinto L. Dental caries experience and molar-incisor hypomineralization. Acta Odontol Scand. 2013 Jan;71(3-4):870-6. doi: 10.3109/00016357.2012.734412.

22. Subramaniam P, Gupta T, Sharma A. Prevalence of molar incisor hypomineralization in 7-9-year-old children of Bengaluru City, India. Contemp Clin Dent. 2016 Jan/Mar;7(1):11-5. doi: 10.4103/0976-237X.177091.

23. Alaluusua S, Lukinmaa L, Vartiainen T, Partanen M, Torppa J, Tuomisto J. Polychlorinated dibenzo-pdioxins and dibenzofurans via mother's milk may cause developmental defects in the child's teeth. Environ Toxicol Pharmacol. 1996 May;1(3):193-7. doi:10.1016/1382-6689(96)00007-5. 
24. Garot E, Rouas P, d'Incau E, Lenoir N, Manton D, Couture C. Mineral dentisty of hypomineralized and sound enamel. Bull Group Int Sci Stomatol Odontol. 2016;53(1):33-6

25. Oyedele TA, Folayan MO, Oziegbe EO. Hypomineralized second primary molars: prevalence, pattern and associated co-morbidities in 8- to 10-year-old children in Ile-lfe, Nigeria. BMC Oral Health. 2016;16(1):65. doi:10.1186/s12903-016-0225-9.

26. Buchgraber B, Kqiku L, Ebeleseder KA. Molar incisor hypomineralization: proportion and severity in primary public school children in Graz, Austria. Clin. Oral Investig. Berlin. 2017 Mar;22(2):757-62. doi: 10.1007/s00784-017-2150-y.

27. Wuollet E, Laisi S, Salmela E, Ess A, Alaluusua S. Molar-incisor hypomineralization and the association with childhood illnesses and antibiotics in a group of Finnish children. Acta Odontol Scand. 2016 May;74(5):1-7. doi: 10.3109/00016357.2016.1172342.

28. Faustino-Silva DD, Rocha AF, da Rocha BS, Stein C. Use of antibiotics in early childhood and dental enamel defects in 6- to 12-year-old children in primary health care. Acta Odontol Latinoam. 2020;33(1):6-13

29. Lopes-Fatturi A, Menezes JVNB, Fraiz FC, Assunção LRDS, de Souza JF. Systemic exposures associated with hypomineralized primary second molars. Pediatr Dent. 2019;41(5):364-70.

30. Bullio Fragelli CM, Jeremias F, Feltrin de Souza J, Paschoal MA, de Cássia Loiola Cordeiro R, Santos-Pinto L. Longitudinal evaluation of the structural integrity of teeth affected by molar incisor hypomineralization. Caries Res. 2015;49(4):378-83. doi: 10.1159/000380858.

31. Temilola OD, Folayan MO, Oyedele T. The prevalence and pattern of deciduous molar hypomineralization and molar-incisor hypomineralization in children from a suburban population in Nigeria. BMC Oral Health. 2015 Jun;15(1):73-9. doi: 10.1186/s12903-015-0059-x. 